\title{
Deiktische Praktiken: Zwischen Interaktion und Grammatik
}

Anja Stukenbrock (Uni Jena)

In der 1983 unter dem Titel „The Interaction Order" erschienenen Rede, die Erving Goffman als Präsident der American Sociological Association kurz vor seinem Tod verfasst hat, etabliert er nachdrücklich die face-to-faceInteraktion als eigenständigen Untersuchungsgegenstand und betont den zentralen Stellenwert leiblicher Kopräsenz und wechselseitiger Wahrnehmung für die Ausgestaltung unserer interaktiver Praktiken.

Goffmans Einsichten in die menschliche Interaktion sind von der Linguistik noch längst nicht ausgeschöpft. Im Rekurs auf sein Erbe möchte ich die Frage danach, "What sorts of animals are to be found in the interactional zoo? What plants in this particular garden?" (Goffman: The Interaction Order: 6) im Sinne der Interaktionalen Linguistik als Frage nach „Sprachstrukturen als Ressourcen der ,Rede-in-der-Interaktion“" (Selting/Couper-Kuhlen 2000: 78) stellen. Theoretischen und empirischen Ankerpunkt bildet die Deixis als phylo- und ontogenetisch (vgl. Tomasello 2003, 2008) herausgehobene Schnittstelle zwischen Interaktion und Grammatik, zwischen Sprache, menschlichem Körper, Objekten, Wahrnehmung und Raum. Anhand von empirischen Beispielen aus einem breit angelegten Videokorpus werden systematisch die transsituativen Gemeinsamkeiten und Unterschiede deiktischer Praktiken dargelegt, die Beteiligte in der Kommunikation von Angesicht zu Angesicht bei der demonstratio ad oculos (dem Zeigen auf Sichtbares, Bühler 1934/65) und der Deixis am Phantasma (dem Zeigen auf Unsichtbares, Bühler 1934/65) verwenden. Die Detailanalysen werden in einen übergreifenden theoretischen Rahmen integriert, der Deixis als situierte, die interaktiven, kognitiven und perzeptorischen Ressourcen aller Beteiligten mobilisierende Praxis gemeinsamer Aufmerksamkeitsfokussierung begreift (Stukenbrock 2015). 